\title{
Resonant Far- to Near-Field Channeling in Synergetic Multiscale Antennas
}

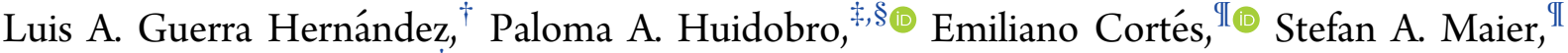 \\ and Alejandro Fainstein $* \dagger \odot$ \\ ${ }^{\dagger}$ Centro Atómico Bariloche e Instituto Balseiro, San Carlos de Bariloche, 8400 Río Negro, Argentina \\ ${ }^{\ddagger}$ The Blackett Laboratory, Department of Physics, Imperial College London, London SW7 2AZ, United Kingdom \\ ${ }^{\text {II }}$ Chair in Hybrid Nanosystems, Nanoinstitute Munich, Faculty of Physics, Ludwig-Maximilians-Universität München, \\ Königinstrasse 10, 80539 München, Germany
}

\begin{abstract}
Enhancing light-molecule interactions requires the efficient transfer of energy between the laboratory macroscale and the molecule nanoscale. Multiscale designs have been proposed as a means to efficiently connect these two worlds. Metallic sphere-segment void (SSV) cavities constitute plasmonic substrates in which light wavelength scale cavity-like modes and nanoscale roughness operate in conjunction as a multiscale antenna to provide larger surface-enhanced Raman scattering efficiency than the two mechanisms considered separately. We study the selective resonant coupling to cavity modes with different spatial distributions in SSV arrays with tailored nanoscale roughness. Cavity modes that are spatially more confined to the surface are demonstrated to lead to more efficient channeling of energy from the far to the near field, a synergy that scales with the degree of roughness. Finiteelement modeling of the spatially varying local fields in rough SSV arrays allows for a microscopic description of the results, opening promising paths for the design of spatially and spectrally optimized multiscale antennas for efficient sensing with far- to near-field channeling of light.
\end{abstract}

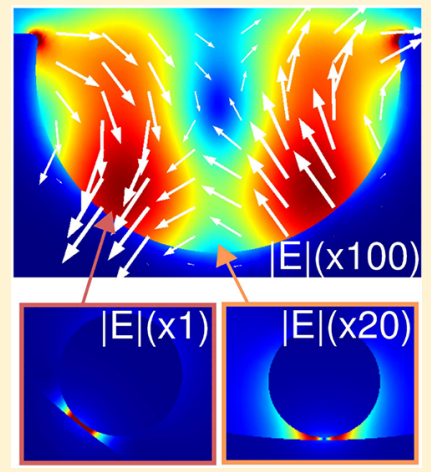

KEYWORDS: plasmonic substrates, sphere-segment void arrays, roughness, multiscale antennas, surface-enhanced Raman spectroscopy, sensing

E fficient coupling of light to molecules for sensing requires strongly enhanced near fields where the molecules reside. ${ }^{1}$ An example of this, with significant practical applications, is surface-enhanced Raman scattering (SERS). In SERS the measured intensities typically scale with the fourth power of the light electric field, thus evidencing the importance of accessing large near-field enhancements. Several ways to achieve this goal have been explored, showing that the most efficient antennas that perform this task are nanoscale plasmonic structures that provide strongly enhanced near fields. ${ }^{2-4}$ These structures by themselves have the disadvantage of producing large near-field amplification at the expense of small cross sections for far-field coupling. On the other hand, strong far-field coupling is better accomplished through antennas with sizes at the scale of the light wavelength (submicrometer size), at the expense of having relatively small light field enhancements, and the maxima of these fields not being necessarily well tuned spatially to efficiently couple with molecules at surfaces. So-called critical coupling schemes, e.g., exploiting the Kretschmann configuration ${ }^{5}$ or using tailored Tamm mode resonators, ${ }^{6,7}$ are an additional resource to optimize the coupling from the far field into these devices.

Briefly then, subwavelength-size nanoscale structures provide large near-field enhancement but reduced coupling to the far field. Conversely, wavelength-size antennas optimize this latter coupling, but their channeling of energy to molecules located at the surface is typically poor. Synergetic multiscale antennas can provide the answer to this conundrum, by efficiently channeling light from the macroscale of the laser source to the molecular scale. Different approaches to this problem have been recently proposed..$^{8-14}$ The conceptual interest of cascading the enhancement of the local and radiated electromagnetic field by the multiscale electromagnetic coupling of hierarchical structures has been recently reviewed in the context of electromagnetic theories of surface-enhanced Raman spectroscopy. ${ }^{15}$ Sphere-segment void (SSV) cavities offer strong and uniform electromagnetic enhancement associated with the excitation of cavity-like surface plasmon resonances tunable across the UV-vis-near IR range, with a facile large-scale fabrication process. ${ }^{16-18}$ We have recently highlighted the relevance of roughness in these parabolic-like antennas. ${ }^{10}$ In fact, upon close structural examination, a considerable departure from the idealized smooth spherical cavity shape is observed, with the presence of an important degree of nanoscale surface roughness intrinsically related to the used $\mathrm{Au}$ electrochemical deposition process. It was concluded from this investigation that both roughness features and cavity-like modes operate in conjunction as a synergetic

Received: January 23, 2019

Published: May 13, 2019 
multiscale antenna to provide larger SERS efficiency than the two mechanisms (cavity confinement or roughness) considered separately. ${ }^{8-10}$ Quite notably, ideal void cavities with surface roughness strongly reduced by thermal annealing showed strongly diminished SERS enhancement. The main role of the ordered cavity structure was thus concluded to be an increase of the local density of states near rough surface features owing to the cavity plasmonic field enhancement, thus optimizing the coupling of far-field radiation to the roughnessinduced locally enhanced near fields. ${ }^{10}$ With these conclusions in mind, one could envisage that for efficient sensing resonant modes should be preferentially exploited that increase their projection onto the surface of the antenna, as compared to others more confined within the empty space of the spheresegment void cavities. It is the purpose of this investigation to address this fundamental question.

Sphere-segment void cavities display a series of cavity-like modes with different spatial distribution, resembling P-, D-, and F-like atomic states. ${ }^{17,19-21}$ Our previous work relied on the coupling of laser light to the fundamental P-like states of $600 \mathrm{~nm}$ diameter cavities, to demonstrate the synergetic performance of roughness and cavity resonances in these multiscale antennas. ${ }^{10}$ However, P-states turn out to be mostly localized at the center of the voids, so that the coupling to molecules immobilized on the surface is not optimized. In fact, their projection to the surface relies mostly on the truncation of the structure that makes it depart from an ideal spherical cavity. ${ }^{20}$ On the contrary, D, F, and additional higher-order modes tend to localize their field closer to the surface and thus should be more efficient for the channeling of energy from the far to the near field for enhanced light-molecule coupling. ${ }^{22}$

In this paper we study these effects by resonant selective coupling to different spatial modes in SSV arrays of tailored nanoscale roughness. For this purpose structures with cavities of large diameter are studied $(800 \mathrm{~nm})$, in which light can couple to both $\mathrm{P}$ - and D-like modes at $\sim 650 \mathrm{~nm}$ laser excitation. Excitation with $\mathrm{Ar}-\mathrm{Kr} 647 \mathrm{~nm}$ laser light is chosen for additional electronic resonance tuned to metal-to-molecule charge transfer transitions. ${ }^{23}$ The selective coupling to P- or alternatively D-like modes is accomplished by tuning the SSV resonance modes through their degree of truncation. ${ }^{24} \mathrm{We}$ demonstrate that indeed coupling to D-like modes provides markedly larger SERS signals from probe molecules than the coupling to P-like states and that this enhancement scales with the degree of roughness. Finite-element modeling of the spatially varying local fields in rough SSV arrays is presented for a microscopic description of the results.

\section{RESULTS AND DISCUSSION}

Sphere-Segment Void Plasmonic Modes. Surface plasmon polaritons (SPPs) can show a variety of modes depending on the surface geometry and excitation conditions. ${ }^{25}$ At planar interfaces the accessible modes are propagating surface plasmon polaritons (PSPPs), which cannot be coupled to light unless a phase-matching method is introduced. For this purpose a glass prism is commonly used (Kretschmann configuration). Patterning of the surface, e.g., with a grating, can lead to PSPP excitation due to the extra momentum gained through the scattering of the incident optical field. Such structures support so-called Bragg modes, which can also be understood as a dispersion relation folding of planar metallic interface SPPs. In finite size structures wavevector is not conserved, and thus light can couple in addition with localized surface plasmon polaritons (LSPPs) confined to these structures. ${ }^{3,4}$ In the context of truncated (SSV) cavity plasmonic substrates, LSPP modes correspond to the Mie kind of modes that are localized in the region of the cavities, and SPP modes refer to the Bragg kind of modes that propagate on the top surface, particularly observed for small ( $t$ $\approx 0)$ and large $(t \approx 1)$ values of the truncation parameter $t$. Here the truncation parameter is defined as $t=h / 2 R$, with $h$ the structure height and $R$ the cavity radius (see the inset of Figure 1(a)). These two kinds of modes mix when their

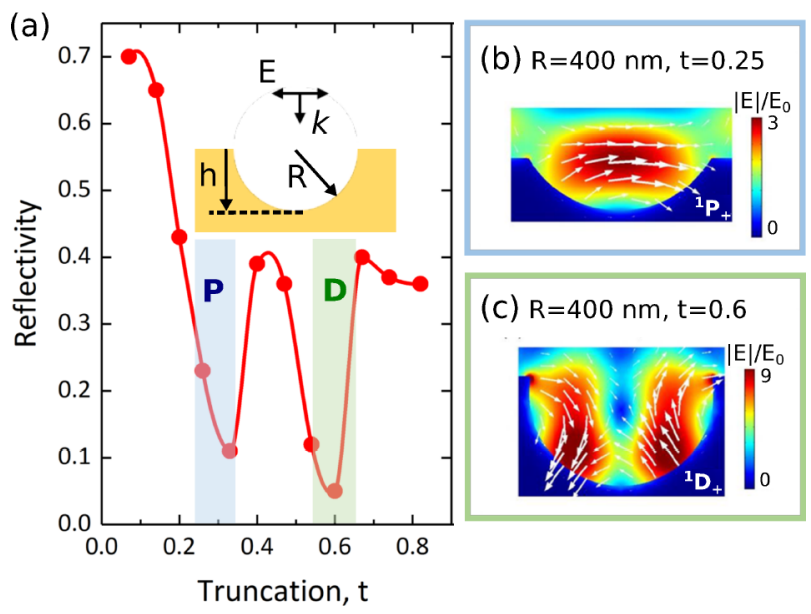

Figure 1. (a) Reflectivity measured at $\theta=25^{\circ}$ incidence angle and $650 \mathrm{~nm}$ for $R=400 \mathrm{~nm}$ cavities as a function of the cavity truncation parameter $t=h / 2 R$. Two modes can be clearly identified (P, D). The inset defines the parameters and the light incidence configuration used in the modeling. (b, c) Calculated mode profiles of $R=400 \mathrm{~nm}$ cavities at normal incidence and around the wavelength of $\sim 650 \mathrm{~nm}$ for two different values of $t$. The cavity with $t=0.25$ (b) shows a $1 \mathrm{P}_{+}$ mode, and the cavity with $t=0.6$ (c) a $1 \mathrm{D}_{+}$mode.

energies coincide (the relative detuning can be changed both by varying the truncation parameter and by changing the cavity diameter), but otherwise their separate nature can be well identified for mostly all situations. Coupling to the two kinds of modes, Mie type (LSPP) and Bragg type (SPP), from the far field is possible in the truncated SSV substrates. Previous work has shown that Bragg-type modes are not efficient for SERS, the largest Raman cross sections being observable by resonantly coupling to the Mie kind of modes. ${ }^{24}$

The resonant wavelength of Mie-like modes of SSV substrates can be tuned in two ways, either by changing the diameter of the voids (larger cavities sustain longer resonant wavelengths) or by changing the degree of truncation of the cavities. A dispersion toward shorter resonant wavelengths is indeed observed for smaller values of the cavity truncation parameter $t$. This latter procedure is experimentally more convenient, because one single graded substrate can be fabricated with the flexibility to probe different resonant conditions. ${ }^{23}$ We self-assembled a monolayer of 4-mercaptobenzoic acid (4-MBA), which is a well-known Raman probe, and used an excitation wavelength of $650 \mathrm{~nm}$, which allowed in previous investigations Raman signals of high intensity. ${ }^{23,24}$ For the previous SERS study of the synergetic contribution of cavity plasmons and nanoscale roughness in SSV substrates reported in ref 10, cavities of $600 \mathrm{~nm}$ diameter were used that only allowed with laser excitation at $\sim 650 \mathrm{~nm}$ coupling to Plike Mie modes, irrespective of the truncation parameter. To 
allow for a comparison study of the relative efficiency of different Mie modes at $\sim 650 \mathrm{~nm}$ excitation wavelength, a shift of the cavity resonances toward the NIR is required. For this purpose we opted for larger cavities of $800 \mathrm{~nm}$ diameter, for which tuning to both $\mathrm{P}$ - and $\mathrm{D}$-like Mie modes is possible at $650 \mathrm{~nm} .^{24}$ This occurs at intermediate values of the truncation parameter $t$, a region that is characterized by high-quality structures (typically defects increase for electrochemical deposition when the structure height $h$ approaches the void diameter $2 R$ and the cavities are closed). ${ }^{24}$

Reflectivity measurements at $\theta=25^{\circ}$ incidence angle were performed to identify the plasmon cavity modes of the studied $800 \mathrm{~nm}$ diameter SSV antennas. Figure 1(a) shows the reflectivity at $650 \mathrm{~nm}$ as a function of the cavity truncation parameter $t$. Two local minima are identified due to the resonant coupling to the $1 \mathrm{P}_{+}$and $1 \mathrm{D}_{+}$Mie-like modes, respectively. Electromagnetic modeling of the optical response of the substrates allows not only confirming this identification but also characterizing the distinctive spatial distribution of the excited SSV localized modes. In fact, the cavity with $t=0.25$ shows at $\sim 650 \mathrm{~nm}$ a $1 \mathrm{P}_{+}$mode (see Figure $1(\mathrm{~b})$ ), while the cavity with $t=0.6$ displays a $1 \mathrm{D}_{+}$mode (shown in Figure 1(c)). ${ }^{19}$ These calculated values for the truncation $t$ at which resonance is attained at $\sim 650 \mathrm{~nm}$ match reasonably well with the experimental data in Figure 1(a). The modeling also shows that the higher order $1 \mathrm{D}_{+}$mode is more intense close to the surface, while the $1 \mathrm{P}_{+}$mode is mostly confined within the empty space of the sphere-segment void antennas. It is this spatial characteristic of the $1 \mathrm{D}_{+}$mode that will be exploited in this work to channel far-field radiation to the nanoscale surface roughness to more efficiently couple to SERS probe molecules bound to the surface.

Synergetic Multiscale SSV Antennas. As demonstrated previously, ${ }^{10}$ SSV arrays present stronger SERS enhancement factors when both nanoscale surface roughness and plasmon modes resulting from the patterned structure act synergetically. Cavity modes allow a good coupling to the far field, providing an enhanced and highly confined light electric field in the region of the voids. Roughness in turn captures this energy and channels it to the molecules at the surface through the plasmonic response of nanoscale protuberances of high curvature. $^{26-28}$ As mentioned above, inspection of Figure 1 evidences that this channeling should be more effective for Dthan for P-like modes. To test these ideas, three kinds of 800 nm diameter SSV substrates were produced: (i) as prepared, (ii) electrochemically roughened, and (iii) thermally annealed to remove residual fabrication roughness. All substrates were graded to display truncation values from $t=0$ to $t=1$ and were tested to confirm equivalent reflectivity responses after preparation and prior to the electrochemical roughening or the annealing procedures. ${ }^{10}$ SEM images of these substrates are shown in the top panels of Figure 2. Intrinsic roughness of typical size $\sim 50 \mathrm{~nm}$ is evident in the as-grown structures. This roughness is almost fully removed by annealing while conserving the segment-sphere void architecture of the substrates and is modified by additional electrochemical processing, again maintaining the overall cavity structure essentially unaltered. ${ }^{10}$ The experiments were performed in the electrochemically processed cavities with a fixed low additional roughness level (a measured increase of area by a factor around 3 ), which already attains a large degree of SERS enhancement and does not expose the cavities to potential damage. The latter was assessed by characterization using imaging

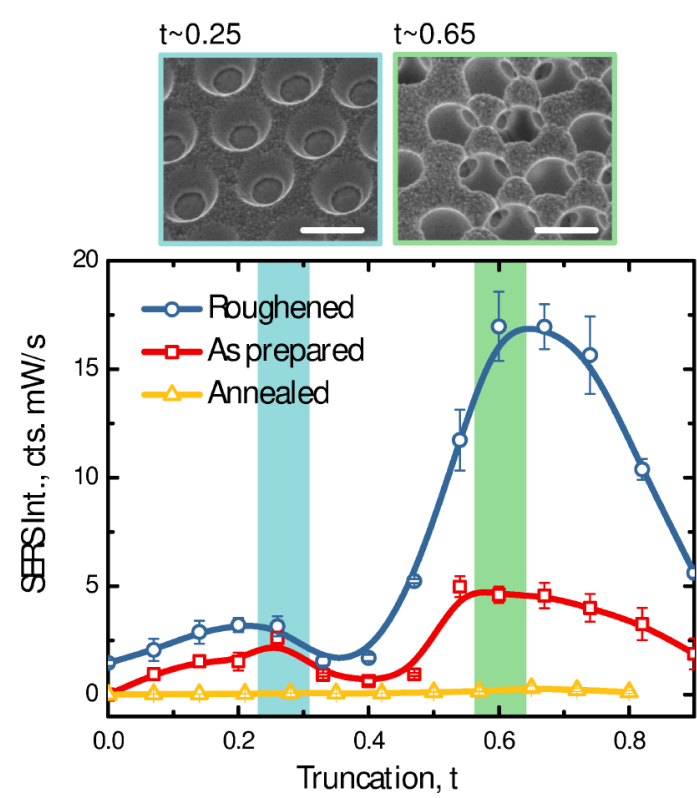

Figure 2. Top panels: Representative high-resolution SEM images of $R=400 \mathrm{~nm}$ Au SSV arrays of truncation parameters $t \approx 0.25$ and $t \approx$ 0.65 , corresponding to structures for which light of $650 \mathrm{~nm}$ couples to P- and D-like modes, respectively. Scale bars are $1 \mu \mathrm{m}$. Bottom panel: SERS intensity of the 4-MBA $1076 \mathrm{~cm}^{-1}$ band as a function of truncation $(t)$, excited at $647 \mathrm{~nm}$ incident light.

techniques (SEM and STM) and reflectivity measurements to check that the Mie-like plasmon modes remain essentially unaltered (except eventually for some small shift). The STM studies demonstrated that the number and typical size of the surface nanostructures are changed by the roughening process withrespect to the as-grown sample (the number is increased and the average size is decreased), but otherwise no spatial inhomogeneity was observed. ${ }^{10}$

Electronically resonant SERRS (surface-enhanced resonant Raman scattering) experiments were performed in order to quantify the field enhancement of the plasmonic substrates. As the SERRS probe molecule we used 4-MBA. This molecule forms well-ordered self-assembled monolayers on $\mathrm{Au}$ with reproducible coverage. ${ }^{29}$ It is a nonresonant Raman molecule in solution, with electronic transitions in the UV, but previous investigations have shown that this and similar thiol-bound molecules develop a resonant electronic ligand-to-metal transition around $\sim 650 \mathrm{~nm}$ when covalently attached to either $\mathrm{Au}$ or Ag. ${ }^{23}$ The bottom panel in Figure 2 shows the Raman intensity of the $1076 \mathrm{~cm}^{-1}$ band of $\mathrm{MBA}$ as a function of the truncation parameter $t$ for the as-grown (square symbols), roughened (circles), and annealed (triangles) SSV substrates, obtained with resonant laser excitation at $647 \mathrm{~nm}$. Notably, SERRS enhancement, and hence near-field enhancement, occurs particularly when resonant coupling to the Mie-like cavity modes of the SSV substrates is achieved. The experiments further demonstrate that the roughened substrate SERS signal strongly increases by $\sim 4$ times in comparison with as-prepared samples. In contrast, annealed structures show almost no detectable signals. Enhancement through roughening and signal quenching by annealing is accomplished while conserving the overall SERRS landscape characterized by enhanced signals when coupling occurs through either the resonant P- or D-like SSV modes. This clearly proves the importance of the interplay of the localized surface nanoscale 
features together with the far-field LSPP antennas in the enhancement of the electric field at the proximity of the molecules. Relevant to this work, the results in Figure 2 prove that, as envisaged, $\mathrm{D}_{+}$modes provide a larger enhancement of the SERS signal (a factor of $\sim 5-6$ ) than $\mathrm{P}_{+}$modes, arguably due to optimized channeling of energy to the surface of the voids. To provide further confirmation of this hypothesis in what follows, we present finite element modeling of the electromagnetic response of the roughened SSV cavities.

Modeling of Surface Electric Field Enhancement in Roughened SSV. Modeling roughness on SSV structures with a finite element method has a huge computational cost due to the diverse length scales at play. In fact, the roughnessassociated nanoscale features need a high spatial discretization that must coexist with the (microscale) void surface structure. An effective model that intends to grasp the main physical ingredients of the problem was thus implemented to simulate the effect of roughness in the near field of truncated $\mathrm{Au}$ cavities. Conceptually, the following features need to be included in the model: (i) the truncated SSV parabolic-like antennas displaying the P- and D-like plasmonic cavity modes, (ii) nanoscale plasmonic resonators that simulate roughness and can be placed at different spatial locations at the surface of these antennas, and (iii) an energy tuning knob for these latter resonators, in the understanding that, similar to the role of hotspots, the SERS process will select those local resonances that are degenerate with the ( $\mathrm{P}$ or $\mathrm{D})$ Mie-like cavity modes of the SSV substrate.

F. M. Huang and co-workers discussed the coupled plasmonic resonator comprising an SSV cavity and a surfacelocalized nanoparticle. ${ }^{8,9}$ They showed that such a hybrid structure behaves quite similarly to a pair of coupled nanoparticles, with field enhancement even larger than this paradigmatic system. Interestingly, as an idealized resonator it contains the required characteristics and degrees of freedom to model the basic features of rough SSV substrates described above, namely, (i) the SSV cavities with their Mie-like localized modes, (ii) the metallic nanoparticle leading to a local gap resonance that can be located anywhere at the SSV surface, and (iii) either the cavity diameter/degree of truncation, the nanoparticle size/shape, or the nanoparticle-cavity surface distance as different ways to spectrally tune the local near-field resonance. On the basis of these considerations, we model the rough cavities with these gap plasmon resonances, with structures consisting of a spherical $\mathrm{Au}$ nanoparticle situated close to the surface of a truncated SSV cavity. We monitor the spectral dependence of the light electric-field amplitude associated with the gap resonance of the nanoparticle-cavity coupled system when a plane wave of a given wavelength impinges on the structure. To study the channeling of light from the far to the near field, this is done for different spatial locations of the gap resonance, particularly where the electric fields associated with the Mie P- or D-like modes have their maxima (see Figure 3).

We first discuss how to select the proper gap resonance so that it is spectrally tuned to the cavity Mie-like modes. Figure 3 (a) shows the calculated resonance wavelength of the $1 \mathrm{P}_{+}$, $1 \mathrm{P}_{-}, 1 \mathrm{D}_{+}$, and $1 \mathrm{D}_{-}$Mie-type modes of a truncated $(t=0.6)$ empty (i.e., no nanoparticle included) Au SSV cavity as a function of the cavity radius. As expected (and verified in the experiments), the resonance wavelength of these cavity modes blue-shifts when reducing the radius, and D-like modes appear at smaller wavelengths (higher energies) when compared to (a)

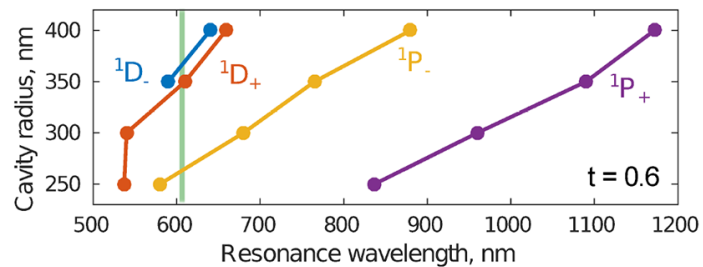

(b)

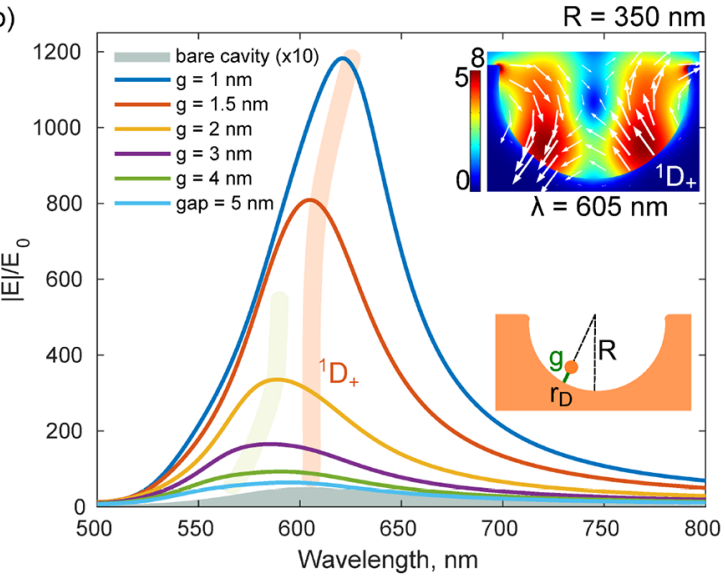

(c)

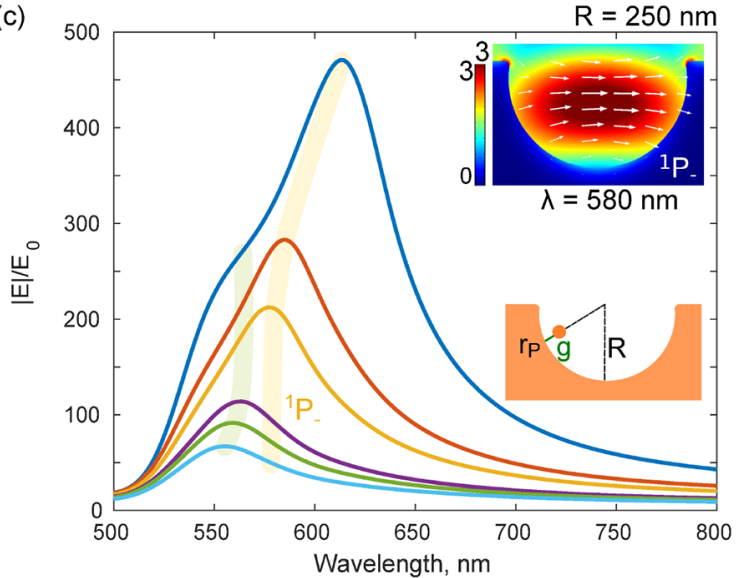

Figure 3. Modeling rough cavities with gap plasmon resonances. (a) Mode spectrum dependence on cavity size for cavities with $t=0.6$. (b) We use an $R=350 \mathrm{~nm}$ cavity to model the interaction with the $\mathrm{D}$ modes $\left(1 \mathrm{D}_{+}\right.$mode at $605 \mathrm{~nm}$ as shown in the inset panel). The figure shows the spectral dependence of the local field enhancement at the mid point of the gap between a $60 \mathrm{~nm}$ nanoparticle and the cavity surface for different gap sizes ranging from 1 to $5 \mathrm{~nm}$. (c) Same as (b) but for an $R=250 \mathrm{~nm}$ cavity to model the interaction with the $\mathrm{P}$ modes $\left(1 \mathrm{P}_{-}\right.$at $580 \mathrm{~nm}$ as shown in the inset panel). For each cavity the nanoparticle is placed where the field in the empty cavity is maximum (positions $r_{\mathrm{D}}$ and $r_{\mathrm{P}}$, see the insets in (b) and (c), respectively). Panel (b) also includes with a shaded curve the spectral response of the field enhancement at $r_{\mathrm{D}}$ and at a distance $g=1.5 \mathrm{~nm}$ from the surface for the bare cavity (i.e., without nanoparticle), to emphasize the strong enhancement induced by the presence of the nanoparticle-cavity gap plasmon.

the P-like modes. The different response obtained by coupling to the two studied P- and D-like Mie modes was modeled by changing the cavity radius for a fixed truncation, instead of changing the truncation parameter (as done in the experiments). As is clear from this figure, by scanning the cavity radius at a given wavelength, one can go through a resonance with the different Mie-like modes. Similarly to the experiments we choose as probing wavelength $600 \mathrm{~nm}$ (highlighted with 
the shaded thick line in Figure 3(a)). We consider two different situations, namely, resonance with a P-like mode (observed for a cavity radius of $R \approx 250 \mathrm{~nm}$ ) and resonance with a $\mathrm{D}$-like mode $(R \approx 350 \mathrm{~nm})$. To tune the gap resonance close to the probe wavelength of $600 \mathrm{~nm}$, we chose as a starting point a spherical nanoparticle of $60 \mathrm{~nm}$ diameter that is expected to have its bare plasmon resonance around 550 nm. ${ }^{8,9}$ The nanoparticle-surface gap separation is then varied to further red-shift the resonance down to $\sim 600 \mathrm{~nm}$. This is shown for both the case of the $R=250 \mathrm{~nm}$ and $R=350 \mathrm{~nm}$ cavities in Figure 3(b) and (c). Data are presented for gap separation $g$ varied between 5 and $1 \mathrm{~nm}$. The curves show the spectral dependence of the field enhancement at the midpoint between the nanoparticle and the surface, as a function of the gap separation $g$, for the nanoparticle located at an azimuthal angle such that the coupling is maximum to the D- (panel (b)) or P-like (panel (c)) modes, respectively. The red-shift of the local resonance as the gap distance decreases is clear, evidencing an anticrossing behavior that is consistent with a strong coupling between this local resonance and the Mie-like cavity modes. Note that panel (b) in Figure 3 also includes with a shaded curve the spectral response of the field enhancement at $r_{\mathrm{D}}$ and at a distance $g=1.5 \mathrm{~nm}$ from the surface evaluated for the bare cavity (i.e., without nanoparticle). Comparison of this and the other curves in the panel emphasizes the strong enhancement induced by the presence of the nanoparticle-cavity gap plasmon. On the basis of these results and to define a local gap resonance at $\sim 605 \mathrm{~nm}$ we chose $g=1.5 \mathrm{~nm}$ for the spatial dependence study presented next.

Figure 4(a) shows the case of the D-like modes, while Figure 4(b) concentrates on the P-like case. For each case, the nanoparticle was placed at an azimuthal angle for which either the field of the corresponding Mie-like mode in the empty cavity was largest (labeled as $r_{\mathrm{D}}$ and $r_{\mathrm{P}}$ in Figure 4, respectively) or at the bottom of the void where the Mie modes have a small projection $\left(r_{0}\right)$. For $r_{\mathrm{D}}$ and $r_{\mathrm{P}}$ the curves represent the spectral dependence of the near-field enhancement at the mid point between the $60 \mathrm{~nm}$ diameter nanoparticle and the cavity surface, where the field is maximum. At the bottom of the voids $\left(r_{0}\right)$ the fields have a node at the midpoint of the gap, and thus we chose to show the field evaluated at the displaced position where it is largest (see in Figure 4 the insets presenting the spatial distribution of the fields for each considered case). Several features of these curves can be highlighted: (i) irrespective of the considered resonant excitation condition, tuned with the $\mathrm{D}$ - or P-like Mie modes, the presence of the nanoparticle completely modifies the field spatial distribution, transferring the maximum field from the cavity void to the near field located at the nanoparticle-cavity gap (which behaves as a hot-spot); (ii) with the gap-plasmon tuned to the corresponding Mie mode, the resonances of the multiscale synergetic antennas are optimized so that the Mie modes are responsible for efficiently harvesting the energy from the far to the near field; and (iii), the field enhancement for the D-like mode is approximately double of that obtained for the P-like mode excitation. This can be understood as due to the spatial distribution of the corresponding Mie-like cavity mode, which for the P-like mode is concentrated out of the surface and toward the center of the SSV. This factor of 2 implies a larger SERS enhancement for coupling through the D-like mode, which for molecules precisely located at the maximum of the gap hot-spot could be
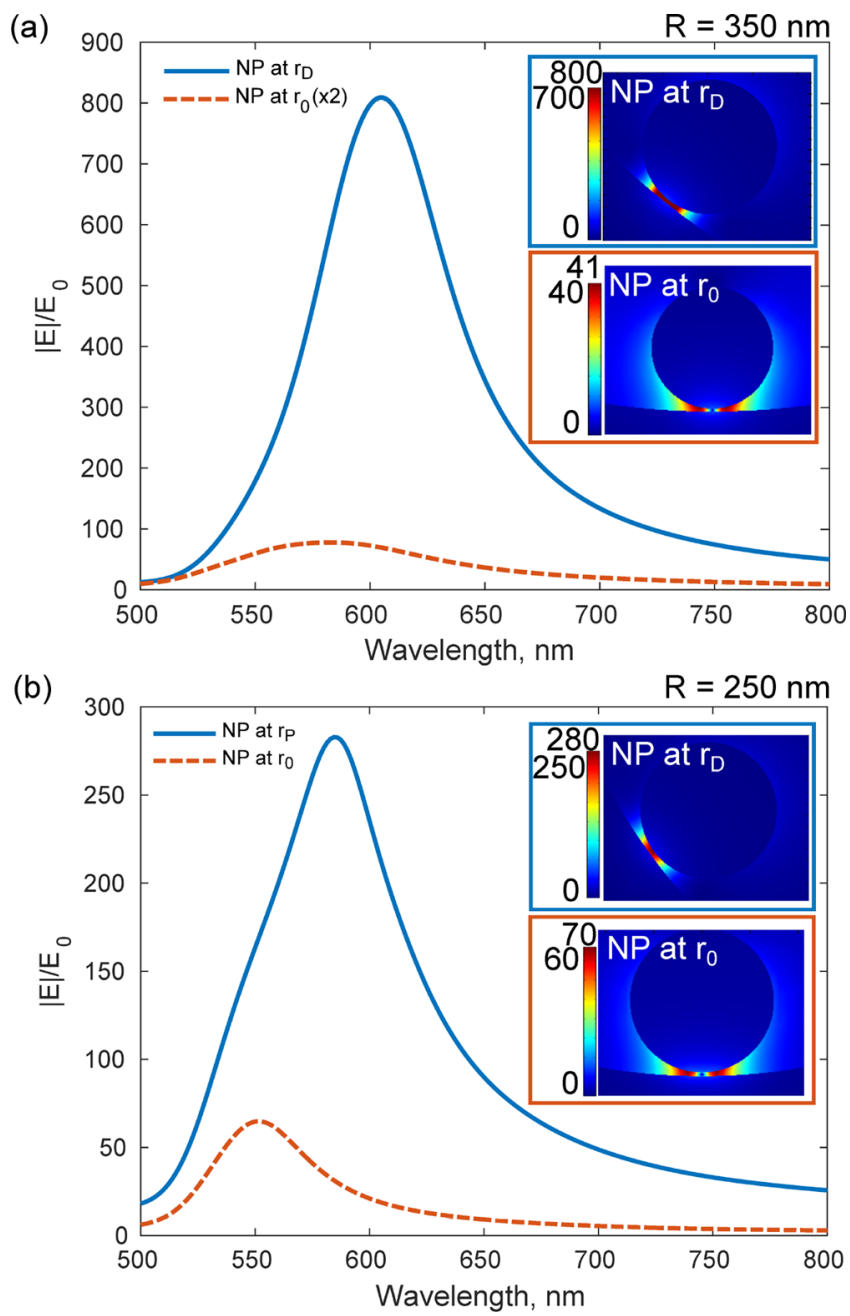

Figure 4. Spatial dependence of far- to near-field channeling. Mode spectral dependence on gap plasmon location for SSVs of truncation $t$ $=0.6$, with $R=350 \mathrm{~nm}$ (a) and $R=250 \mathrm{~nm}$ (b). The spectra correspond to the maximum field in the gap region for a nanoparticle-surface separation $g=1.5 \mathrm{~nm}$. For each cavity the nanoparticle is placed where the field of the corresponding Mie mode in the empty cavity is maximum and also for comparison at the bottom of the void where the projection of the mode is small. Examples of field distribution with $600 \mathrm{~nm}$ resonant excitation and for the nanoparticles localized at the different positions (either $r_{\mathrm{D}}, r_{\mathrm{P}}$, or $r_{0}$ ) are presented in the insets (see the text for more details).

as high as a factor of $\sim 16$ (i.e., $2^{4}$ ). Note that this is somewhat larger than the relative enhancement observed in the experiments, which amounts to $\sim 5-6$, as observed in Figure 2. It is however a reasonable agreement taking into account the fact that laser and Stokes photons cannot be both perfectly tuned at resonance (such double resonance is a requirement for the dependence of the enhancement on the fourth power of the fields). In addition, in the experiment there is an averaging over different spatial locations that do not take advantage of the maximum enhancement accessible. Indeed, note that we are considering that the places of the cavity producing a high SERS signal are the ones where the nanoscale roughness can resonantly couple with the Mie cavity modes. Other nonresonant spots with different shapes would not contribute significantly to the total SERS signal, as this scales approximately with the fourth power of the electric field. Note also that in all cases the field enhancement at the bottom 
of the void is almost an order of magnitude smaller, evidencing the relevance of the spatial distribution of the Mie modes for the efficient channeling of light from the far to the near field.

\section{CONCLUSIONS AND PERSPECTIVES}

We have shown that multiscale antennas comprising Mie modes of SSV antennas for efficient harvesting of far-field light and nanoscale roughness for its channeling to the near field where molecules are immobilized can be engineered and synergetically exploited for the efficient sensing of molecules. Furthermore, this type of multiscale substrate may also find interesting applications in plasmonic photocatalysis, behaving as a new type of antenna-reactor system. ${ }^{30,31}$ Detailed electromagnetic modeling of the proposed system mimicking the effect of roughness through hybrid nanoparticle-cavity gap-plasmon resonances allowed for the understanding of the spatial and spectral response of the far- to near-field channeling. D-like modes with larger optical density at the surface are shown to be more efficient than P-like modes, which have a larger projection into the open space of the cavities of the truncated SSV substrates. F and higher-order modes that have been previously identified in SSV substrates to have an increasing projection to the surface could be exploited to extend the concept demonstrated here. Calculations demonstrate that the synergetic action of far-field collection and near-field channeling select those hot-spots that reside at the spatial location where the Mie modes are preferentially distributed and that are spectrally degenerate with the corresponding Mie mode. We believe that our approach highlights interesting strategies for the conception of tailored plasmonic substrates optimized to both efficiently collect light from the far field and translate its energy to the surface where molecules reside, maintaining at the same time the harvesting potential of microscale structures and the enhancement efficiency of nanoscale hot-spots.

\section{METHODS}

Fabrication of SSV Arrays. Samples were fabricated using nanosphere lithography with ordered spheres of $400 \mathrm{~nm}$ radius. A stepwise variation of truncation height allows the tracking and identification of specific plasmon modes as implemented. ${ }^{19}$ SSV arrays were produced according to the procedure detailed in refs 16 and 32. In this method polystyrene spheres dispersed in a $1 \%$ water solution (Duke Scientific) are introduced between a glass substrate coated with a $100 \mathrm{~nm}$ gold film (Platypus) and a clean glass slide, with a gap of about $300 \mu \mathrm{m}$. The gold film is previously immersed in a $1 \mathrm{mM}$ cysteamine ethanolic solution overnight to improve the adsorption of polystyrene spheres. During the drying process in an incubation chamber, a sweeping meniscus forms along the substrate, pulling the spheres toward the substrate and into a close-packed hexagonal monolayer. This selfassembly of the template is followed by electrodeposition of $\mathrm{Au}$ from a gold salt solution (TG-25 RTU, Technic Inc.), with a deposition rate of $2.5 \mathrm{mC} / \mathrm{min}$. The sample is mounted on a micrometer stage, allowing progressive withdrawing from the solution during the deposition by steps of $500 \mu \mathrm{m}$, yielding discrete levels of truncation parameters for a given sample. ${ }^{23}$ The polystyrene spheres are eventually removed via sonication in a sequence of solvents (dimethylformamide, acetone, ethanol, and water) for 2 min per step.
Annealing and Roughening of SSV Arrays. By heating the sample at $300{ }^{\circ} \mathrm{C}$ for $60 \mathrm{~min}$ we obtained a reduction of nanoscale surface roughness, while preserving the global structure of the SSV arrays. SSV arrays were fabricated as previously described, and cyclic voltammetry in $0.5 \mathrm{M} \mathrm{H}_{2} \mathrm{SO}_{4}$ solution was used to check the decrease in exposed area. Thermal annealing was performed in a homemade conventional oven, with a heating rate set to $10{ }^{\circ} \mathrm{C} \mathrm{min}^{-1}$. After reaching the desired temperature $\left(300{ }^{\circ} \mathrm{C}\right)$ we checked that temperature fluctuations were no greater than $10{ }^{\circ} \mathrm{C}$.

Substrate Characterization. The characterization of the plasmonic modes was performed with reflectivity measurements in a variable-angle spectroscopic ellipsometer (Wollam WVASE32). A $100 \mu \mathrm{m}$ circular spot was focused on the samples with a numerical aperture of $\sim 0.02$.

SEM images were recorded with a FEI field-emission gun and a Nova NANO-SEM 230 operating at $10 \mathrm{kV}$ and both at normal incidence and with a tilt angle of $45 \mathrm{deg}$.

SERS measurements were performed using a triple-stage Raman spectrometer (Horiba Jobin-Yvon T64000) operating in subtractive mode and equipped with a liquid-nitrogencooled charge-coupled device. The excitation was done at a fixed wavelength of $647 \mathrm{~nm}$ and $\sim 20 \mathrm{~mW}$ power of an $\mathrm{Ar}: \mathrm{Kr}$ laser focused onto a $\sim 50 \mu \mathrm{m}$ spot. The position on the sample was manually controlled, and the Raman signals were collected in a backscattering configuration with a collection lens of focal length $10 \mathrm{~cm}$. The entrance slit of the spectrometer was kept at $200 \mu \mathrm{m}$, as the Raman peaks for the metal-adsorbed molecule are relatively broad and do not require high spectral resolution. Typical acquisition times were $120 \mathrm{~s}$ for the as-grown SSV substrates and $30 \mathrm{~s}$ for roughened cavities, presenting enhanced signals.

Theoretical Modeling of Roughness. Local roughness of gold cavities has been modeled by considering nanometersized gaps between the cavity surface and spherical gold nanoparticles. We study the effect of roughness in this structures and compare its effect for the $\mathrm{D}$ and $\mathrm{P}$ modes. This is done by characterizing the electric field enhancement at the midpoint of the gap between the surface and the particle as the gap size is reduced down to $1.0 \mathrm{~nm}$. The EM response of the system was calculated by sending a plane wave at normal incidence at isolated cavities. Empty cavities were first used to track the spectral location of different Mie modes, and two cavity sizes were selected such that the D (cavity radius 350 $\mathrm{nm}$ ) and $\mathrm{P}$ modes (radius $250 \mathrm{~nm}$ ) were tuned to the experimental wavelength $(\sim 600 \mathrm{~nm})$. Then, cavities with particles were simulated to model the effect of roughness. The particle was placed in the area close to the surface where field enhancement was maximum, different in both cavities. Calculations of the EM field were done using the finite element method. ${ }^{33}$ Field plots shown in Figures 3 and 4 present the electric field enhancement ( $|E|$ normalized to the incident amplitude) at a cross section of the three-dimensional cavities defined by the plane of incidence of the wave (electric field lying along the horizontal axis). The dielectric permittivity of the metal in the simulations was taken from Johnson and Christie's data. ${ }^{34}$

\section{AUTHOR INFORMATION}

\section{Corresponding Author}

*E-mail: afains@cab.cnea.gov.ar. Phone: +54 (0)116 772 7947. Fax: +54 (0)2944 445102 . 


\section{ORCID $\odot$}

Paloma A. Huidobro: 0000-0002-7968-5158

Emiliano Cortés: 0000-0001-8248-4165

Alejandro Fainstein: 0000-0001-7875-3609

\section{Present Address}

${ }^{\S}$ P.A.H.: Departamento de Física Teórica de la Materia Condensada and Condensed Matter Physics Center (IFIMAC), Universidad Autónoma de Madrid, E-28049 Madrid, Spain

\section{Notes}

The authors declare no competing financial interest.

\section{ACKNOWLEDGMENTS}

L.A.G. and A.F. thank ANPCyT for support through grants PICT-2013-2047, PICT-2014-0196, and PICT-2015-1591. S.A.M. acknowledges the DFG Cluster of Excellence NIM (Nanoscience Initiative Munich), the Bavarian SOLTEC (Solar Energies Go Hybrid) program, ONR Global, and the Lee-Lucas Chair in Physics. P.A.H. acknowledges funding from the Gordon and Betty Moore Foundation. A.F. is also a member of CONICET.

\section{REFERENCES}

(1) Chikkaraddy, R.; de Nijs, B.; Benz, F.; Barrow, S. J.; Scherman, O. A.; Rosta, E.; Demetriadou, A.; Fox, P.; Hess, O.; Baumberg, J. J. Single-molecule strong coupling at room temperature in plasmonic nanocavities. Nature 2016, 535, 127.

(2) Stiles, P. L.; Dieringer, J. A.; Shah, N. C.; Van Duyne, R. P. Surface-enhanced Raman spectroscopy. Annu. Rev. Anal. Chem. 2008, $1,601-626$.

(3) Halas, N. J.; Lal, S.; Chang, W.-S.; Link, S.; Nordlander, P. Plasmons in strongly coupled metallic nanostructures. Chem. Rev. 2011, 111, 3913-3961.

(4) Giannini, V.; Fernández-Domínguez, A. I.; Heck, S. C.; Maier, S. A. Plasmonic nanoantennas: fundamentals and their use in controlling the radiative properties of nanoemitters. Chem. Rev. 2011, 111, 38883912.

(5) Kretschmann, E.; Raether, H. Radiative decay of non radiative surface plasmons excited by light. Z. Naturforsch., A: Phys. Sci. 1968, $23,2135-2136$

(6) Auguié, B.; Bruchhausen, A.; Fainstein, A. Critical coupling to Tamm plasmons. J. Opt. 2015, 17, 035003.

(7) Tsurimaki, Y.; Tong, J. K.; Boriskin, V. N.; Semenov, A.; Ayzatsky, M. I.; Machekhin, Y. P.; Chen, G.; Boriskina, S. V. Topological engineering of interfacial optical Tamm states for highly sensitive near-singular-phase optical detection. ACS Photonics 2018, 5, 929-938.

(8) Huang, F. M.; Wilding, D.; Speed, J. D.; Russell, A. E.; Bartlett, P. N.; Baumberg, J. J. Dressing Plasmons in Particle-in-Cavity Architectures. Nano Lett. 2011, 11, 1221-1226.

(9) Speed, J. D.; Johnson, R. P.; Hugall, J. T.; Lal, N. N.; Bartlett, P. N.; Baumberg, J. J.; Russell, A. E. SERS from molecules bridging the gap of particle-in-cavity structures. Chem. Commun. 2011, 47, 63356337.

(10) Guerra Hernández, L. A.; Daza Millone, M. A.; Cortés, E.; Castez, M. F.; Auguié, B.; Vela, M. E.; Salvarezza, R. C.; Fainstein, A. Synergetic Light-Harvesting and Near-Field Enhancement in Multiscale Patterned Gold Substrates. ACS Photonics 2015, 2, 1355-1365.

(11) Trügler, A.; Tinguely, J.-C.; Krenn, J. R.; Hohenau, A.; Hohenester, U. Influence of surface roughness on the optical properties of plasmonic nanoparticles. Phys. Rev. B: Condens. Matter Mater. Phys. 2011, 83, 081412.

(12) Li, K.; Stockman, M. I.; Bergman, D. J. Self-similar chain of metal nanospheres as an efficient nanolens. Phys. Rev. Lett. 2003, 91, 227402 .
(13) Peyskens, F.; Wuytens, P.; Raza, A.; Van Dorpe, P.; Baets, R. Waveguide excitation and collection of surface-enhanced Raman scattering from a single plasmonic antenna. Nanophotonics 2018, 7, 1299.

(14) Verma, P. Tip-enhanced Raman spectroscopy: technique and recent advances. Chem. Rev. 2017, 117, 6447-6466.

(15) Ding, S.-Y.; You, E.-M.; Tian, Z.-Q.; Moskovits, M. Electromagnetic theories of surface-enhanced Raman spectroscopy. Chem. Soc. Rev. 2017, 46, 4042-4076.

(16) Bartlett, P. N.; Birkin, P. R.; Ghanem, M. A. Electrochemical deposition of macroporous platinum, palladium and cobalt films using polystyrene latex sphere templates. Chem. Commun. 2000, 16711672.

(17) Perney, N.; García de Abajo, F.; Baumberg, J.; Tang, A.; Netti, M.; Charlton, M.; Zoorob, M. Tuning localized plasmon cavities for optimized surface-enhanced Raman scattering. Phys. Rev. B: Condens. Matter Mater. Phys. 2007, 76, DOI: 10.1103/PhysRevB.76.035426.

(18) Cortés, E.; Tognalli, N. G.; Fainstein, A.; Vela, M. E.; Salvarezza, R. C. Ag-modified Au nanocavity SERS substrates. Phys. Chem. Chem. Phys. 2009, 11, 7469.

(19) Kelf, T.; Sugawara, Y.; Cole, R.; Baumberg, J.; Abdelsalam, M.; Cintra, S.; Mahajan, S.; Russell, A.; Bartlett, P. Localized and delocalized plasmons in metallic nanovoids. Phys. Rev. B: Condens. Matter Mater. Phys. 2006, 74, DOI: 10.1103/PhysRevB.74.245415.

(20) Cole, R. M.; Baumberg, J. J.; García de Abajo, F. J.; Mahajan, S.; Abdelsalam, M.; Bartlett, P. N. Understanding plasmons in nanoscale voids. Nano Lett. 2007, 7, 2094-2100.

(21) Lacharmoise, P.; Tognalli, N.; Goñi, A.; Alonso, M.; Fainstein, A.; Cole, R.; Baumberg, J.; Garcia de Abajo, J.; Bartlett, P. Imaging optical near fields at metallic nanoscale voids. Phys. Rev. B: Condens. Matter Mater. Phys. 2008, 78, DOI: 10.1103/PhysRevB.78.125410.

(22) Lordan, F.; Rice, J. H.; Jose, B.; Forster, R. J.; Keyes, T. E. Site selective surface enhanced Raman on nanostructured cavities. Appl. Phys. Lett. 2011, 99, 033104.

(23) Tognalli, N. G.; Cortés, E.; Hernández-Nieves, A. D.; Carro, P.; Usaj, G.; Balseiro, C. A.; Vela, M. E.; Salvarezza, R. C.; Fainstein, A. From Single to Multiple Ag-Layer Modification of Au Nanocavity Substrates: A Tunable Probe of the Chemical Surface-Enhanced Raman Scattering Mechanism. ACS Nano 2011, 5, 5433-5443.

(24) Tognalli, N. G.; Fainstein, A.; Calvo, E. J.; Abdelsalam, M.; Bartlett, P. N. Incident Wavelength Resolved Resonant SERS on Au Sphere Segment Void (SSV) Arrays. J. Phys. Chem. C 2012, 116, $3414-3420$

(25) Maier, S. A. Plasmonics, Fundamentals and Applications; Springer: Berlin, 2007.

(26) Trügler, A.; Tinguely, J.-C.; Jakopic, G.; Hohenester, U.; Krenn, J. R.; Hohenau, A. Near-field and SERS enhancement from rough plasmonic nanoparticles. Phys. Rev. B: Condens. Matter Mater. Phys. 2014, 89, 165409.

(27) Macias, G.; Alba, M.; Marsal, L. F.; Mihi, A. Surface roughness boosts the SERS performance of imprinted plasmonic architectures. J. Mater. Chem. C 2016, 4, 3970-3975.

(28) Lee, C.; Robertson, C. S.; Nguyen, A. H.; Kahraman, M.; Wachsmann-Hogiu, S. Thickness of a metallic film, in addition to its roughness, plays a significant role in SERS activity. Sci. Rep. 2015, 5, 11644.

(29) Pensa, E.; Rubert, A.; Benitez, G.; Carro, P.; Orive, A. G.; Creus, A. H.; Salvarezza, R.; Vericat, C. Are 4-mercaptobenzoic acid self assembled monolayers on $\mathrm{Au}(111)$ a suitable system to test adatom models? J. Phys. Chem. C 2012, 116, 25765-25771.

(30) Li, K.; Hogan, N. J.; Kale, M. J.; Halas, N. J.; Nordlander, P.; Christopher, P. Balancing near-field enhancement, absorption, and scattering for effective antenna-reactor plasmonic photocatalysis. Nano Lett. 2017, 17, 3710-3717.

(31) Zhang, C.; Zhao, H.; Zhou, L.; Schlather, A. E.; Dong, L.; McClain, M. J.; Swearer, D. F.; Nordlander, P.; Halas, N. J. Al -Pd nanodisk heterodimers as antenna-reactor photocatalysts. Nano Lett. 2016, 16, 6677-6682. 
(32) Bartlett, P. N.; Baumberg, J. J.; Coyle, S.; Abdelsalam, M. E. Optical properties of nanostructured metal films. Faraday Discuss. 2004, 125, 117.

(33) Comsol Multiphysics software, https://www.comsol.com/ comsol-multiphysics.

(34) Johnson, P. B.; Christy, R.-W. Optical constants of the noble metals. Phys. Rev. B 1972, 6, 4370. 